\title{
Hyperarousal Scale: Italian Cultural Validation, Age and Gender Differences in a Nonclinical Population
}

\author{
Antonio Bruno ${ }^{1, *(\mathbb{D}}$, Amelia Rizzo ${ }^{2}\left(\mathbb{D}\right.$, Maria Rosaria Anna Muscatello ${ }^{1}\left(\mathbb{D}\right.$, Laura Celebre ${ }^{1}(\mathbb{D}$, \\ Maria Catena Silvestri ${ }^{2}$, Rocco Antonio Zoccali ${ }^{1}$ and Carmela Mento ${ }^{1}$ (D) \\ 1 Department of Biomedical and Dental Sciences and Morphofunctional Imaging, University of Messina, \\ Via Consolare Valeria 1, Contesse, 98125 Messina, Italy; mmuscatello@unime.it (M.R.A.M.); \\ lallacelebre@gmail.com (L.C.); zoccali@unime.it (R.A.Z.); cmento@unime.it (C.M.) \\ 2 Psychiatry Unit, Polyclinic Hospital University of Messina, Via Consolare Valeria 1, Contesse, 98125 Messina, \\ Italy; amrizzo@unime.it (A.R.); mariacatenasilvestri89@gmail.com (M.C.S.) \\ * Correspondence: antonio.bruno@unime.it; Tel.: +39-090-22212092; Fax: +39-090-695136
}

Received: 28 December 2019; Accepted: 10 February 2020; Published: 12 February 2020

\begin{abstract}
Objectives. Studies on hyperarousal have increasingly developed in the last decade. Nevertheless, there are still very few valid measures of hyperarousal. The aim of the study is to verify the psychometric properties of the Italian version of the Hyperarousal Scale (H-Scale), in order to provide researchers with a valid measure for the target population. Method. The questionnaire was translated, back-translated, pre-tested, and cross-culturally adapted. Subsequently, the Italian version of the H-Scale, the Anxiety Sensitivity Index (ASI-3) and the Health Survey Questionnaire (SF-36) were administered to 982 adults, 456 males and 526 females, aged from 18 to 80 years ( $M=35.61 \pm 12.47)$. Results. Cronbach's alpha of the translated H-Scale was 0.81. Furthermore, positive correlations with the ASI-3 and negative correlations with the SF-36 emerged. The H-Scale is also sensitive to catch age and gender differences. Conclusions. The Italian version of the H-Scale demonstrated good reliability and validity. Its sufficient discriminative and evaluative psychometric properties provide the theoretical evidence for further application in evidence-based research studies.
\end{abstract}

Keywords: hyperarousal; H-Scale; validation; gender differences

\section{Introduction}

Hyperarousal is an abnormal state of increased responsiveness to stimuli marked by various physiological and psychological symptoms, such as elevated heart rate and respiration and increased levels of alertness and anxiety. This state of amplified sensitivity is characterized by constant hypervigilance, difficulty in relaxing, increased anger or irritability, reckless or self-destructive behaviors, and increased startle response [1].

The DSM-5 resumes the hyperarousal state in Criterion E of post-traumatic stress disorder (PTSD), describing changes in the state of arousal, which started or worsened following the experience of a traumatic event, such as (a) irritability or aggressive behavior, (b) impulsive or self-destructive behavior, (c) feeling constantly "on guard" or like danger is lurking around every corner (or hypervigilance), (d) heightened startle response, (e) difficulty concentrating, and (f) sleep problems [2]. Research shows that among PTSD symptoms, hyperarousal is probably most closely related to impulsive behaviors; intense anxiety and discomfort associated with hyperarousal may lead subjects to look for immediate relief by acting impulsively, without considering possible outcomes. Furthermore, the experience of negative affective states characterized by high levels of arousal, along with emotion dysregulation, which has been found to be heightened among individuals with PTSD, are underlying 
mechanism of several specific impulsive behaviors, such as substance abuse, reckless driving, or drinking excessively [3].

Difficulty falling asleep is one of the core symptoms of hyperarousal. As a consequence, sleep deprivation can negatively affect mood, concentration, and the ability to cope with and manage stress, with potential long-term physical health consequences [4]. Given the major impact of hyperarousal on sleep quantity and quality, it is not surprising that its measurement appeared for the first time in clinical studies on insomnia and in research focused on neurological and neurophysiological aspects of hyperactivation. Regestein and colleagues [5] were the first to conduct an event-related potential (ERP) study in patients with insomnia, finding that the 26-item Hyperarousal Scale (H-Scale), empirically designed for measuring daytime alertness, accurately distinguished insomnia patients from controls. The insomnia group was characterized by higher alertness often accompanied by increased emotion, and furtherly confirmed by electrophysiological evidence of daytime arousal. Literature showed that the H-Scale scores were higher in insomniacs and correlated with the severity of insomnia [6] and that, in general, all sleep disorder groups had increased total hyperarousal scores. These self-report findings suggest that insomnia subjects may generally show more vulnerability to environmental requests [7].

In the past few years, it has become increasingly clear that hyperarousal can be a chronic condition interacting with many other psychiatric disorders, and that experiencing hyperarousal along with other symptoms can be tied to both physical and mental health. Hammad and colleagues [8] hypothesized a connection between hyperarousal and somatization, probably based on altered information processing; findings suggested that hyperarousal explained a substantial part of the variance (about 20\%) of the construct of somatization.

Hyperarousal has also been related to depression. Szelenberger and Niemcewicz [9] found correlations between hyperarousal and depression scores, demonstrating relationships with the cognitive and affective aspects of depression; moreover, depressive symptoms and night eating were key factors related to insomnia [10]. Higher levels of self-reported arousal were associated with distress from interpersonal problems; individuals with insomnia who reported more distress from interpersonal problems tended to have more severe insomnia and cognitive pre-sleep arousal, possibly due to rumination [11].

Since women are more likely than men to suffer from PTSD [12] and major depression (MD) [13], a possible moderating role of hyperarousal on vulnerability to such disorders has been taken into account in order to explain gender differences in stress-related disorders. Preclinical data reported sex differences in stress response systems, as documented by differences in brain arousal centers and cellular and molecular mechanisms, such as receptor trafficking, cell signaling, hormone release, and peptide expression, highlighting sex differences that can be linked to increased arousal responses to stress in females compared with males [14].

Moreover, the correlation between hyperarousal and higher levels of negative and positive emotionality was found in both genders [15] but, when compared exclusively on the hyperarousal trait, women generally reported higher scores [16].

The construct of hyperarousal is thought to represent a specific cluster of PTSD symptoms, but it also comprises and contributes to rumination [17], sleep disturbances [18], and hyper-activation symptoms, which are more frequently observed in female patients [19].

Despite the increasing research interest on hyperarousal, to date there are very few measures assessing the hyperarousal trait. Potential candidate questionnaires include the H-Scale used in studies of insomnia patients [5,7]; nevertheless, data on psychometric properties of this questionnaire are still scarce [20].

The H-Scale, also known as HAS [21,22], measures information processing, tendencies to introspection, thinking about feelings, intense responses to unexpected stimuli, and other behaviors that putatively involve cortical arousal. Higher scores correspond to a heightened state of arousal; in the original publication, a total score of $\geq 40$ had a sensitivity of $90 \%$ and specificity of $100 \%$ for identifying subjects with primary insomnia versus controls [23]. Nevertheless, Khassawneh et al. [24] 
found that subjects with sleep disorders obtained a mean score $\geq 29$ on H-Scale, whereas controls obtained a mean score $<26$. The H-Scale is assumed to assess the effect of both cognitive and somatic hyperarousal [25], but a standardized cut-off point is not available; the unavailability of instruments has even brought some researchers to use the self-report H-Scale combined with the hyperarousal items by the PTSD Checklist and the Clinician-Administered PTSD Scale (CAPS) [26].

Hyperarousal is also assessed by a subscale from the Impact of Event Scale-Revised (IES-R) [27,28], a measure of subjective distress caused by a specific traumatic event. IES-R is composed of 22 items across three subscales: intrusion, avoidance, and hyperarousal. The total score is obtained by summing the items, with higher scores indicating higher levels of traumatic response.

Furthermore, hyperarousal can be measured in children by the Physiological Hyperarousal Scale for Children, a specific instrument developed by Laurent, Catanzaro, and Joiner [29] which does not fit the adult population.

In conclusion, the most frequently used questionnaire assessing hyperarousal levels is the H-Scale [30], which is available only in English and Swedish [16].

To our knowledge, there are no valid and reliable measures for evaluating the hyperarousal trait in the Italian population. This factor greatly limits studies and research that, as we have seen, include important clinical aspects that are transversal to various mental disorders, making it difficult to compare data and restraining the generalizability of results for the reference population.

Hence, there is a need for valid and reliable measures for assessing the hyperarousal trait for application in evidence-based research studies. To date, there are no adaptations of the H-Scale for use in the Italian context. The present study aimed to develop and validate an Italian version of the H-Scale in a sample of nonclinical adults, with particular attention towards age and gender differences.

\section{Method}

\subsection{Translation, back Translation, and Cultural Adaptation of the H-Scale}

The H-Scale was translated into Italian by two psychiatrists and two psychologists, all Ph.D., who were well-skilled in English. The first version of the questionnaire was back-translated into English by an English teacher. After back-translation, comparison, and modification of the no-matching items, the final version of the translated scale was formed.

Cultural adaptation of the Italian version was accomplished among university class students. Conceptual equivalence and semantic equivalence were investigated to make a further final revision. Discrepancies emerging from this procedure were discussed until an agreement on a common version was reached.

\subsection{Data Collection and Procedures}

The three psychological instruments-in order H-Scale, the Anxiety Sensitivity Index-3 (ASI-3), and the 36-Item Short-Form Health Survey (SF-36) -were inserted in an online tool panel for data collection (i.e., Google Forms ${ }^{\circledR}$ ) and sent as invitation to participate in the research through institutional mailing lists, posts on social network sites such as Facebook ${ }^{\circledR}$ and Linked-In ${ }^{\circledR}$, other professional mailing lists, and web advertising. The web page remained available for approximately two months, during Summer 2018.

The research method avoided incomplete protocols since the online application did not allow to proceed when one response was left unanswered. The informed consent, which briefly explained the research purposes and guaranteed anonymity, has been indicated in the header of the web page. The consensus was considered valid only when respondents submitted the fully compiled interviews. Answering the items take about 20-30 min, and smartphone use was allowed.

All procedures had been conducted according to the Declaration of Helsinki. The entire study procedure followed the International Test Commission (ITC) guidelines on quality control in scoring, 
test analysis, and reporting of test scores [31]. We reported how we determined all data exclusions, all manipulations, and all measures in the study [32].

\subsection{Participants}

The total study sample consisted of 982 Italian adults, 526 women (53.6\%) and 456 men (46.4\%) aged between 18 and 80 years (Mean $=35.61 \pm 12.47$ ). The whole sample was divided into three subgroups according to age:

young adults, from 18 to 30 years $(\mathrm{N}=447 ; \%=45.5)$;

middle-aged, from 30 to 50 years $(\mathrm{N}=395 ; \%=40.2)$;

older adults, from 51 to 80 years $(\mathrm{N}=140 ; \%=14.3)$.

The educational level was a high-school diploma for $39 \%$ of the subjects $(\mathrm{N}=386)$ and graduation for the remaining $61 \%(\mathrm{~N}=596)$. The gender groups significantly differed in age (females vs males mean age (S.D.) $=36.9$ (12.6) vs $34.4(12.3) ; t=3.216 ; p=0.001)$; moreover, age groups presented significant differences in gender distribution $(\mathrm{X} 2=18.508 ; p<0.0001)$.

\subsection{Instruments}

Socio-demographic and personal information-participants completed a socio-demographic form (gender, age, and level of education).

\subsection{Hyperarousal Scale [5]}

The H-Scale consists of 26 items that assess the hyperarousal behavioral trait on a four-point Likert-type scale coded as $0=$ not at all, $1=$ a little, $2=$ quite a bit, and $3=$ extremely.

The scale produces a Total Summation Score (HSUM); a score of "introspectiveness", i.e., a possible tendency to ruminate, including six items $(4 ; 5 ; 9 ; 11 ; 22 ; 23)$, score range $0-18$; "reactivity", i.e., the "startle response", including three items $(6 ; 12 ; 17)$ score range $0-9$; and "extreme responses" referring to the total number of items checked as "extremely" ranging from 0 to 26 . The higher the total (max. 78), the higher the level of hyperactivation.

\subsection{Anxiety Sensitivity Index-3 (ASI-3) [33]}

The ASI-3, Italian version [34], is an 18-item self-report questionnaire composed of three 6-item subscales:

-Physical Concerns, items 3, 4, 7, 8, 12, 15 (sample item: "It scares me when my heart beats rapidly");

-Cognitive Concerns, items 2, 5, 10, 14, 16, 18 (sample item: "When my mind goes blank, I worry there is something terribly wrong with $\left.\mathrm{me}^{\prime \prime}\right)$;

-Social Concerns, items 1, 6, 9, 11, 13, 17 (sample item: "It scares me when I blush in front of people").

Participants were asked to indicate the extent to which they agreed or disagreed with each item on a 5 -point Likert scale ranging from $0=$ very little to $4=$ very much. Subscales (range $=0-24)$ and total (range $=0-72$ ) scores were calculated by summing relevant items. Nonclinical subjects obtained a mean of $12.19 \pm 9.22$. Finally, higher scores indicated a higher level of anxiety sensitivity. In the present study, ASI-3 achieved an alpha value of 0.90, showing excellent reliability.

\subsection{6-Item Short-Form Health Survey (SF-36) [35]}

For the assessment of the health profile, the SF-36 was used. The scale consists of 36 items subdivided into 8 health scales, i.e., general health $(\mathrm{GH})$, physical functioning $(\mathrm{PF})$, role physical (RP), bodily pain (BP), vitality (VT), role emotional (RE), social function (SF), and mental health (MH). 
Items have different answer options (2, 3, 5, or 6). For several areas, furthermore, the coded score on the evaluation sheet has been recorded, or in other words, reversed, but in some cases also calibrated so lower scores would indicate a worse health-related quality.

In the present study, the raw scores were calculated for each area by summing the scores of the individual items. If more than $50 \%$ of the items in an area were not evaluated, the score for that area was considered missing. The raw scores obtained, to be comparable with each other, were then transformed on a scale from 0 to 100. Higher scores of SF-36 indicated a higher rate of health-related quality of life. In the present study, the SF-36 obtained an alpha value of 0.78 , showing good reliability.

\subsection{Statistical Method}

The Statistical Package for the Social Sciences SPSS v. 16.0 ${ }^{\circledR}$ (IBM, Armonk, NY)was used for data analysis. Student's t-test for independent samples and chi-square tests were performed to evaluate differences among groups on demographic features. To verify the psychometric properties of the translated H-Scale, Pearson's correlation and ANCOVA with Bonferroni post-hoc tests were used; eta-squared statistic was provided to represent the effect size. The significance level for the test was $p<0.05$; additionally, Bonferroni correction was performed for the correlation analyses.

\section{Results}

\subsection{Descriptives}

Table 1 shows the descriptive statistics of the whole sample. The median and the mode resulted around $38.19(\mathrm{SD}=8.89)$ with a mode score of 40.00 . Skewness and kurtosis values suggested that respondents were distributed as a normal/Gaussian curve (see Figure 1). Subjects obtained a minimum of $\mathrm{H}$-sum of $14 / 0$ and a maximum of $67 / 78$, loading the $86 \%$ of the possible maximum score. The percentile cut-offs are also presented in Table 1.

Table 1. Descriptives of the H-Scale in the study sample.

\begin{tabular}{|c|c|c|}
\hline \multicolumn{3}{|c|}{ H-Sum } \\
\hline \multicolumn{2}{|l|}{ Mean } & 38.19 \\
\hline \multicolumn{2}{|l|}{ Median } & 38.00 \\
\hline \multicolumn{2}{|l|}{ Mode } & 40.00 \\
\hline \multicolumn{2}{|c|}{ Std. Deviation } & 8.89 \\
\hline \multicolumn{2}{|c|}{ Skewness } & 0.237 \\
\hline \multicolumn{2}{|c|}{ Std. Error of Skewness } & 0.078 \\
\hline \multicolumn{2}{|c|}{ Kurtosis } & 0.028 \\
\hline \multicolumn{2}{|c|}{ Std. Error of Kurtosis } & 0.156 \\
\hline \multicolumn{2}{|c|}{ Minimum } & 14 \\
\hline \multirow[t]{2}{*}{ Maximum } & & 67 \\
\hline & $25^{\circ}$ & 33.00 \\
\hline \multirow[t]{2}{*}{ Percentiles } & $50^{\circ}$ & 38.00 \\
\hline & $75^{\circ}$ & 44.00 \\
\hline
\end{tabular}




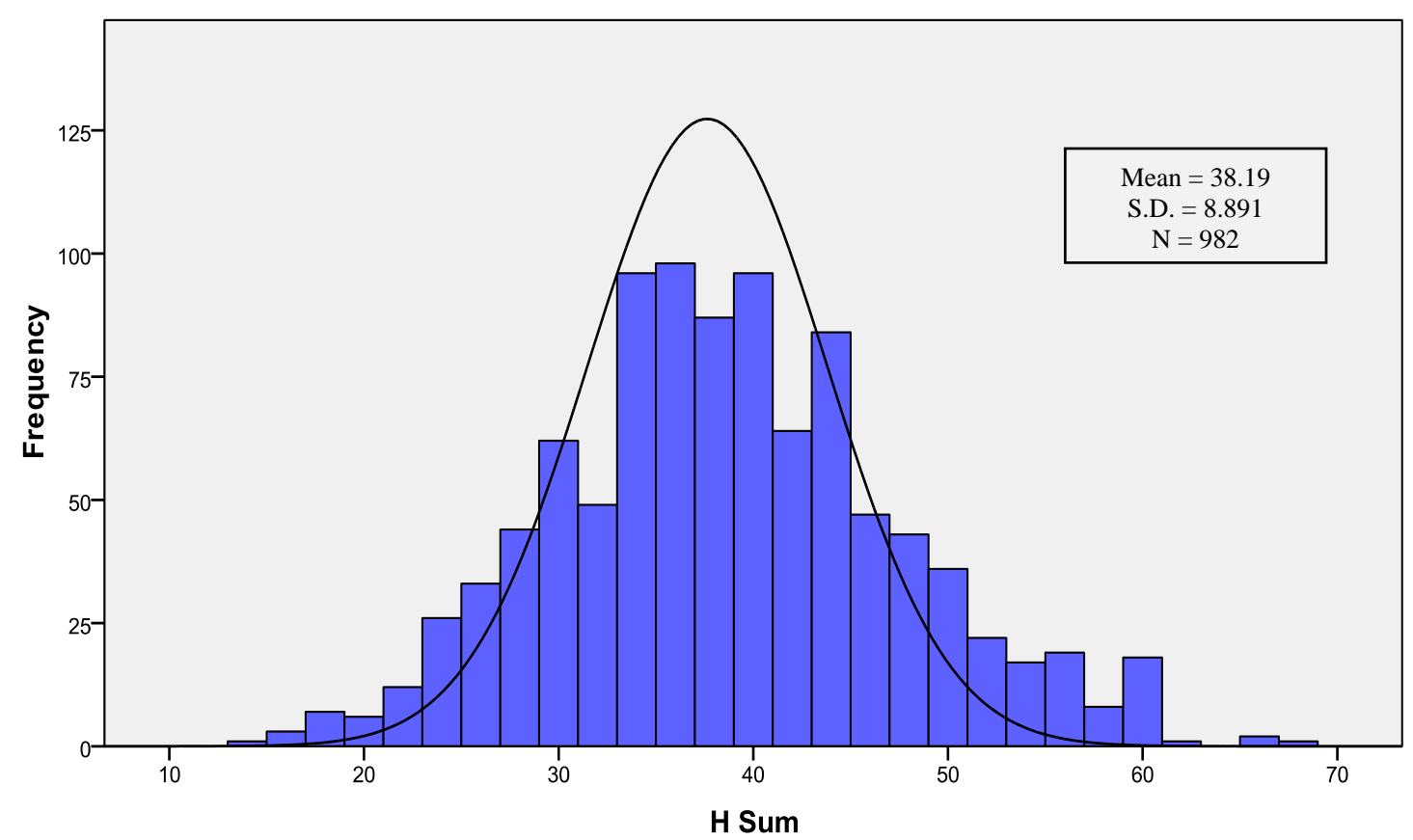

Figure 1. H-Scale sum distribution, histogram with a normal curve.

\subsection{Reliability and Internal Consistency}

To verify the reliability of the scale, all the items were tested with the reliability analysis for the scale. The result indicated that the H-Scale internal consistency was adequate. Cronbach's alpha coefficient was 0.81 , above the recommended 0.70 threshold, and could be slightly improved by deletion of item $1(\alpha=0.82)$. Since the reliability was in both cases very high, item 1 was preserved.

\subsection{Concurrent and Discriminant Validity}

To evaluate the concurrent validity, Pearson's correlation coefficient was performed. We hypothesized positive correlations with anxiety sensitivity and negative correlations with physical and mental health. As can be observed in Table 2, all aspects of anxiety sensitivity (i.e., the physical, cognitive, and social concerns) were positively correlated with all the H-Scale dimensions, such as introspectiveness, reactivity, and extreme response counts. Furthermore, there was a strong correlation even between the H-sum and the ASI-3 total score, which indicated the inter-relation between the constructs of hyperarousal and anxiety sensitivity. When the Bonferroni correction was performed for the number of hypotheses, scores did not change, and correlations between subscales remained statistically significant.

Table 2. H-Scale correlations with ASI-3.

\begin{tabular}{lcccc}
\hline \multicolumn{1}{c}{ H-Scale } & Introspectiveness & Reactivity & Extreme & H-Sum \\
\hline Physical Concern & $0.321 * \S$ & $0.411 * \S$ & $0.305 * \S$ & $0.428 * \S$ \\
Cognitive Concern & $0.470 * \S$ & $0.467 * \S$ & $0.412 * \S$ & $0.546 * \S$ \\
Social Concern & $0.408 * \S$ & $0.437 * \S$ & $0.314 * \S$ & $0.501 * \S$ \\
ASI-3 Total & $0.469 * \S$ & $0.516 * \S$ & $0.403 * \S$ & $0.578 * \S$ \\
\hline
\end{tabular}

${ }^{*} p<0.05 ; \S$ Statistical significance after Bonferroni correction $(\alpha=0.003)$.

To test the discriminant validity, the relationship between hyperarousal and general health, as assessed by the SF-36, was evaluated through Pearson's correlation coefficients. Results showed, as expected, negative correlations (all with a significance level of $p<0.001$ ) among H-Scale subscales 
(introspectiveness, reactiveness, and extreme responses) and total score, and all SF-36 subscales. Furthermore, the absolute correlation coefficients were lower for the discriminant analyses than for the concurrent analyses. Findings indicated that the higher the H-Scale scores (hyper-activation) the lower the general health, including mental health (Table 3).

Table 3. Correlations between H-Scale and SF-36.

\begin{tabular}{lcccc}
\hline \multicolumn{1}{c}{ SF-36 } & \multicolumn{2}{c}{ H-Scale } & H-Sum \\
\hline Change in health status & $-0.110^{*} \S$ & $-0.139^{*} \S$ & $-0.086^{*}$ & $-0.137^{*} \S$ \\
Physical Functioning & $-0.069^{*}$ & $-0.151^{*} \S$ & $-0.151^{*} \S$ & $-0.140^{*} \S$ \\
Role Physical & $-0.203^{*} \S$ & $-0.244^{*} \S$ & $-0.193^{*} \S$ & $-0.266^{*} \S$ \\
Bodily Pain & $-0.197^{*} \S$ & $-0.231^{*} \S$ & $-0.166^{*} \S$ & $-0.253^{*} \S$ \\
Vitality & $-0.393^{*} \S$ & $-0.347^{*} \S$ & $-0.323^{*} \S$ & $-0.469^{*} \S$ \\
Role Emotional & $-0.374^{*} \S$ & $-0.317^{*} \S$ & $-0.290^{*} \S$ & $-0.432^{*} \S$ \\
Social Function & $-0.438^{*} \S$ & $-0.361^{*} \S$ & $-0.378^{*} \S$ & $-0.495^{*} \S$ \\
Mental Health & $-0.465^{*} \S$ & $-0.431^{*} \S$ & $-0.392^{*} \S$ & $-0.533^{*} \S$ \\
General Health & $-0.323^{*} \S$ & $-0.316^{*} \S$ & $-0.268^{*} \S$ & $-0.395^{*} \S$ \\
\hline
\end{tabular}

${ }^{*} p<0.05$; Statistical significance after Bonferroni correction $(\alpha=0.001)$.

When the Bonferroni correction was performed for the number of hypotheses, scores did not change and correlations between subscales remained statistically significant, except for "introspectiveness/physical functioning" and "extreme/change in health status" correlations.

\subsection{Age and Gender Differences}

We performed ANCOVA (with gender as covariate) and a Bonferroni post-hoc test to compare the three groups stratified by age. Age differences are shown in Table 4. Our results suggest that scores of the H-Scale were age-dependent. In particular, the scores of both introspectiveness and reactivity, along with extreme responses and $\mathrm{H}$-sum, tended to decrease with increasing age/maturity. Young adults, compared with middle-aged and older subjects, showed higher scores on "introspectiveness" ( $p<0.0001$; Bonferroni post-hoc test: young adults vs middle-aged: $p<0.0001$; young adults vs older adults: $p<0.0001)$, "extreme response" ( $p<0.0001$; Bonferroni post-hoc test: young adults vs middle-aged: $p=0.003$; young adults vs older adults: $p=0.005)$, and "H-sum" ( $p<0.0001$; Bonferroni post-hoc test: young adults vs middle-aged: $p<0.0001$; young adults vs older adults: $p<0.0001$ ). Furthermore, young adults scored higher than middle-aged subjects $(p<0.0001$; Bonferroni post-hoc test: young adults vs middle-aged: $p<0.0001$ ) on "reactivity" subscale. No significant differences emerged between middle-aged and older subjects. Effect sizes, according to eta-squared, were large in "introspectiveness" and "H-sum", and medium in "reactivity" and "extreme responses" dimensions.

Table 4. Age differences in H-Scale.

\begin{tabular}{|c|c|c|c|c|c|c|c|c|c|}
\hline \multirow{2}{*}{ H-Scale } & \multicolumn{2}{|c|}{ Young adults } & \multicolumn{2}{|c|}{ Middle-aged } & \multicolumn{2}{|c|}{ Older adults } & \multicolumn{2}{|c|}{ ANCOVA } & \multirow[b]{2}{*}{$\eta 2$} \\
\hline & Mean & SD & Mean & SD & Mean & SD & $F(2)$ & Sig. & \\
\hline Introspectiveness & 10.96 & 2.68 & 9.95 & 2.95 & 9.52 & 2.76 & 15.260 & $<0.0001$ & 0.201 \\
\hline Reactivity & 4.02 & 1.74 & 3.47 & 1.93 & 3.66 & 1.79 & 7.329 & $<0.0001$ & 0.139 \\
\hline $\begin{array}{l}\text { Extreme } \\
\text { Responses }\end{array}$ & 3.91 & 3.60 & 3.12 & 3.31 & 2.85 & 3.39 & 6.472 & $<0.0001$ & 0.126 \\
\hline H-Sum & 40.33 & 8.50 & 36.68 & 8.72 & 35.62 & 9.07 & 19.354 & $<0.0001$ & 0.223 \\
\hline
\end{tabular}

Furthermore, we conducted an ANCOVA (with age serving as covariate) to compare scores of the H-Scale between males and females (Table 5). Our results indicated that women showed a higher ruminative tendency as suggested by subscale "introspectiveness" $(p<0.0001)$, and an increased startle 
response as assessed by the items belonging to the "reactivity" subscale $(p=0.004)$. Moreover, women more easily tended to check as positive the "extreme response" $(p<0.0001)$, thus obtaining higher total scores, as compared to men (H-sum $p<0.0001)$. Effect sizes, according to eta-squared, were medium in all explored H-Scale dimensions and total score.

Table 5. Gender differences in H-Scale.

\begin{tabular}{lcccccccc}
\hline \multirow{2}{*}{ H-Scale } & \multicolumn{2}{c}{ Males } & \multicolumn{2}{c}{ Females } & \multicolumn{4}{c}{ ANCOVA } \\
Mean & SD & Mean & SD & F & df & Sig. & $\eta \mathbf{\eta}$ \\
\hline Introspectiveness & 10.08 & 2.82 & 10.58 & 2.88 & 21.631 & 2 & $<0.0001$ & 0.088 \\
Reactivity & 3.61 & 1.90 & 3.87 & 1.78 & 5.563 & 2 & 0.004 & 0.069 \\
Extreme & 3.17 & 3.68 & 3.69 & 3.28 & 8.911 & 2 & $<0.0001$ & 0.074 \\
Responses & 37.20 & 8.92 & 39.05 & 8.77 & 24.512 & 2 & $<0.0001$ & 0.104 \\
H-Sum & & & & & & & &
\end{tabular}

\section{Conclusions}

The present study was aimed to test validity, reliability, and psychometric properties of the Italian translation of the H-Scale in a nonclinical sample of Italian-speaking adults. The concurrent validity was primarily assessed by correlating the H-Scale with the ASI-3. Findings revealed positive correlations between the hyperarousal measure and the Anxiety Sensitivity Index; the strength of correlations indicates that the construct is congruent, but not overloading. Moreover, to further test discriminant and concurrent validity, $\mathrm{H}$-Scale has been correlated with a dissimilar instrument, the SF-36 scale; in this case, negative correlations have been obtained. In other words, hyperarousal was negatively associated with health components: the higher the level of hyperactivity, the lower the health-related quality of life.

In this study, H-Scale reliability has been demonstrated by additional internal consistency analyses, whose results are partially congruent with the previous Swedish validation study [16]. As regards gender differences, we found that women reported higher scores in all $\mathrm{H}$-Scale subscales than men. Literature demonstrated higher levels of hyperarousal-related psychiatric disorders, such as PTSD and $\mathrm{MD}$ among women. According to our results, the hyperarousal trait was more prominent even in a nonclinical sample of women from the general population.

Furthermore, we found that older subjects showed lower scores on the $\mathrm{H}$-scale and $\mathrm{H}$-subscales. This result is in contrast with findings from the Swedish validation study, in which no evidence of age trend was documented; however, it should be noted that the Swedish study had enrolled middle-aged and aged subjects ( 40 years or older). It could be hypothesized that the ability to self-regulate arousal may increase over the years; however, this possible explanation cannot be addressed here, and it probably deserves further studies and/or meta-analyses.

The Italian version of the H-Scale appears to be a reliable and valid self-report instrument for the assessment of hyperarousal behavioral traits, and it can be suitable for clinical research in the Italian population as well as for multi-country studies. Cronbach's alpha showed an acceptable score for the internal consistency of the scale. These findings add further knowledge on the psychometric properties of this questionnaire [20].

Similarly to Buysse et al. [23], we obtained a mean that was also the median value, but a clinical cut-off is still missing. To date, H-Scale has been applied in sleep research, and main results have shown that subjects with insomnia scored significantly higher than controls with no sleep disorders [24]. To the best of our knowledge, this is the first study to investigate the Italian translation of this instrument in a nonclinical population, providing elements for the first acquisition of statistical normative data.

This study has several limitations that should be considered. A major limitation is that, aside from the self-report scale, no objective assessments of hyperarousal have been included. Jawinski et al. [36] used an automated EEG-based algorithm to measure brain arousal in the resting state and demonstrated an association with self-reported daytime sleepiness. In the same vein, EEG results might be compared 
to results of the H-scale. Interestingly, in line with the findings of Szelenberger and Niemcewicz [9], this EEG-based algorithm has been applied in clinical samples with psychiatric diseases believed to have arousal-related pathophysiologies [37-39]. In sum, future studies may further investigate the overlap between subjective scores of the H-Scale and objective measures, both in clinical and nonclinical samples.

Normative data have been recruited in an adequately large sample from the general population, and findings should be verified in clinical samples of patients. As previously reported, the connections between hyperarousal, insomnia, and post-traumatic experiences have been explored. Since hyperarousal symptoms may contribute to the clinical presentation of mood and stress-related disorders, it would be interesting to examine their potential role in other mental disorders, such as personality disorders, obsessive-compulsive disorder, and panic disorders. Furthermore, although there are clearly undeniable advantages of web-based assessments, such as cost reductions if compared with paper-and-pencil methods, the lower probability of receiving socially desirable responses, the immediate availability of data in electronic format, and the elimination of multiple responses, it should be borne in mind that web-based administration may oversample subjects from socially advantaged groups characterized by the availability of electronic devices and high literacy levels.

Beyond the above-mentioned limitations, the Italian version of the H-Scale is a valid and reliable candidate to evaluate the hyperarousal behavioral trait in further research on a range of psychiatric disorders.

Author Contributions: Conceptualization, A.B., R.A.Z., and C.M.; methodology, A.R., and M.R.A.M.; validation, L.C., and M.C.S.; formal analysis, A.B., and A.R.; data curation, L.C., and M.C.S.; writing-original draft preparation, A.R.; writing—review and editing, A.B., and M.R.A.M.; supervision, C.M.; project administration, R.A.Z. All authors have read and agreed to the published version of the manuscript.

Funding: This research received no specific grant from any funding agency in the public, commercial, or not-for-profit sectors.

Conflicts of Interest: The authors declare that there are no financial, general, and institutional conflict of interest regarding the publication of this article.

\section{References}

1. Riemann, D.; Spiegelhalder, K.; Feige, B.; Voderholzer, U.; Berger, M.; Perlis, M.; Nissen, C. The hyperarousal model of insomnia: A review of the concept and its evidence. Sleep Med. Rev. 2010, 14, 19-31. [CrossRef]

2. American Psychiatric Association. Diagnostic and Statistical Manual of Mental Disorders; American Psychiatric Publishing: Arlington, VA, USA, 2013.

3. Weiss, N.H.; Tull, M.T.; Viana, A.G.; Anestis, M.D.; Gratz, K.L. Impulsive behaviors as an emotion regulation strategy: Examining associations between PTSD, emotion dysregulation, and impulsive behaviors among substance dependent inpatients. J. Anxiety Disord. 2012, 26, 453-458. [CrossRef]

4. Kalmbach, D.A.; Cuamatzi-Castelan, A.S.; Tonnu, C.V.; Tran, K.M.; Anderson, J.R.; Roth, T.; Drake, C.L. Hyperarousal and sleep reactivity in insomnia: Current insights. Nat. Sci. Sleep 2018, 10, 193. [CrossRef]

5. Regestein, Q.; Pavlova, M.; Casares, F. Validation of the hyperarousal scale in primary insomnia subjects. Sleep Res. 1996, 25, 344.

6. Niemcewicz, S.; Szelenberger, W.; Skalski, M.; Androsiuk, W.; Piotrowski, T.; Myszka, D.M.; Moskwa, M. Psychophysiological correlates of primary insomnia. Psychiatr. Pol. 2001, 35, 583-591. [PubMed]

7. Pavlova, M.; Berg, O.; Gleason, R.; Walker, F.; Roberts, S.; Regestein, Q. Self-reported hyperarousal traits among insomnia patients. J. Psychosom. Res. 2001, 51, 435-441. [CrossRef]

8. Hammad, M.A.; Barsky, A.J.; Regestein, Q.R. Correlation between somatic sensation inventory scores and hyperarousal scale scores. Psychosomatics 2001, 42, 29-34. [CrossRef]

9. Szelenberger, W.; Niemcewicz, S. Event-related current density in primary insomnia. Acta Neurobiol. Exp. 2001, 61, 299-308.

10. Wołyńczyk-Gmaj, D.; Różańska-Walędziak, A.; Ziemka, S.; Ufnal, M.; Brzezicka, A.; Gmaj, B.; Januszko, P.; Fudalej, S.; Czajkowski, K.; Wojnar, M. Insomnia in pregnancy is associated with depressive symptoms and eating at night. J. Clin. Sleep Med. 2017, 13, 1171-1176. [CrossRef] 
11. Gunn, H.E.; Troxel, W.M.; Hall, M.H.; Buysse, D.J. Interpersonal distress is associated with sleep and arousal in insomnia and good sleepers. J. Psychosom. Res. 2014, 76, 242-248. [CrossRef]

12. Gavranidou, M.; Rosner, R. The weaker sex? Gender and post-traumatic stress disorder. Depress. Anxiety 2003, 17, 130-139. [CrossRef] [PubMed]

13. Kornstein, S.G.; Schatzberg, A.F.; Thase, M.E.; Yonkers, K.A.; McCullough, J.P.; Keitner, G.I.; Gelenberg, A.J.; Ryan, C.E.; Hess, A.L.; Harrison, W.; et al. Gender differences in chronic major and double depression. J. Affect. Disord. 2000, 60, 1-11. [CrossRef]

14. Bangasser, D.A.; Valentino, R.J. Sex differences in stress-related psychiatric disorders: Neurobiological perspectives. Front. Neuroendocrinol. 2014, 35, 303-319. [CrossRef] [PubMed]

15. Hantsoo, L.; Khou, C.S.; White, C.N.; Ong, J.C. Gender and cognitive-emotional factors as predictors of pre-sleep arousal and trait hyperarousal in insomnia. J. Psychosom. Res. 2013, 74, 283-289. [CrossRef] [PubMed]

16. Edéll Gustafsson, U.; Carstensen, J.; Regestein, Q.; Swahn, E.; Svanborg, E. Hyperarousal, depression and quality of life-validity and reliability of the Swedish version of the Hyperarousal Scale. Scand. J. Caring Sci. 2006, 20, 58-67. [CrossRef] [PubMed]

17. Nolen-Hoeksema, S.; Jackson, B. Mediators of the gender difference in rumination. Psychol. Women Q. 2001, 25, 37-47. [CrossRef]

18. Krishnan, V.; Collop, N.A. Gender differences in sleep disorders. Curr. Opin. Pulm. Med. 2006, 12, 383-389. [CrossRef]

19. Parker, G.; Brotchie, H. Gender differences in depression. Int. Rev. Psychiatry 2010, 22, 429-436. [CrossRef]

20. Bakotic, M.; Radosevic-Vidacek, B. State-trait arousal and daytime sleepiness after sleep restriction. Int. J. Psychophysiol. 2013, 88, 164-170. [CrossRef]

21. Guo-Zhong, M.; Xiao-Jiang, J.; Zhi-Qiang, X.; Ying, L.; Heng-Jiang, G.; Ya-Zheng, L.; Dai-Qu, Z.; Rong-Hu, W.; Xu-Jun, L.; Ni-Ka, C.; et al. Effect of dexmedetomidine-induced sleep balance treatment on the chronic refractory primary insomnia patients. J. Basic Appl. Sci. 2014, 10, 102-109.

22. Li, X.; Jiang, X.; Liu, Y.; Lang, Y.; Ye, Y.; Lin, L.; Liu, J.; Zhang, Y. Efficacy of dexmedetomidine with cognitive behavioral therapy for treating chronic insomnia related to conditioned arousal: A randomized controlled trial. Sleep Biol. Rhythm. 2016, 14, 75-85. [CrossRef]

23. Buysse, D.J.; Thompson, W.; Scott, J.; Franzen, P.L.; Germain, A.; Hall, M.; Moul, D.E.; Nofzinger, E.A.; Kupfer, D.J. Daytime symptoms in primary insomnia: A prospective analysis using ecological momentary assessment. Sleep Med. 2007, 8, 198-208. [CrossRef] [PubMed]

24. Khassawneh, B.Y.; Bathgate, C.J.; Tsai, S.C.; Edinger, J.D. Neurocognitive performance in insomnia disorder: The impact of hyperarousal and short sleep duration. J. Sleep Res. 2018, 27, e12747. [CrossRef] [PubMed]

25. Guo, J.; Huang, W.; Tang, C.Y.; Wang, G.L.; Zhang, F.; Wang, L.P. Effect of acupuncture on sleep quality and hyperarousal state in patients with primary insomnia: Study protocol for a randomised controlled trial. BMJ Open 2016, 6, e009594. [CrossRef] [PubMed]

26. Seo, J.; Moore, K.; Gazecki, S.; Pace-Schott, E. F40. In Trauma-Exposed individuals, Self-Reported Hyperarousal Predicts Resting-State Functional Connectivity in Frontocortical and Paralimbic Regions. Biol. Psychiatry 2018, 83, S253. [CrossRef]

27. Asukai, N.; Kato, H.; Kawamura, N.; Kim, Y.; Yamamoto, K.; Kishimoto, J.; Miyake, Y.; Nishizono-Maher, A. Reliabiligy and validity of the Japanese-language version of the impact of event scale-revised (Ies-RJ): Four studies of different traumatic events. J. Nerv. Ment. Dis. 2002, 190, 175-182. [CrossRef]

28. Weiss, D.S. The impact of event scale: Revised. In Cross-Cultural Assessment of Psychological Trauma and PTSD; Springer: Boston, MA, USA, 2007; pp. 219-238.

29. Laurent, J.; Catanzaro, S.J.; Joiner, T.E., Jr. Development and preliminary validation of the physiological hyperarousal scale for children. Psychol. Assess. 2004, 16, 373. [CrossRef]

30. Suh, S.; Song, P.; Kim, H. Assessing Sleep Disorders in the Asian Client. In Guide to Psychological Assessment with Asians; Springer: New York, NY, USA, 2014; pp. 327-346.

31. International Test Commission. ITC guidelines on quality control in scoring, test analysis, and reporting of test scores. Int. J. Test. 2014, 14, 195-217. [CrossRef]

32. Simmons, J.P.; Nelson, L.D.; Simonsohn, U. A 21 Word Solution. SPSP's Dialogue Newsletter. $2012,1-4$. [CrossRef] 
33. Taylor, S.; Zvolensky, M.J.; Cox, B.J.; Deacon, B.; Heimberg, R.G.; Ledley, D.R.; Abramowitz, J.S.; Holaway, R.M.; Sandin, B.; Stewart, S.H.; et al. Robust dimensions of anxiety sensitivity: Development and initial validation of the Anxiety Sensitivity Index-3. Psychol. Assess. 2007, 19, 176-188. [CrossRef]

34. Petrocchi, N.; Tenore, K.; Couyoumdjian, A.; Gragnani, A. The Anxiety Sensitivity Index-3: Factor structure and psychometric properties in Italian clinical and non-clinical samples. BPA Appl. Psychol. Bull. 2014, 62, 53-64.

35. Ware, J.E., Jr.; Sherbourne, C.D. The MOS 36-item short-form health survey (SF-36): I. Conceptual framework and item selection. Med. Care 1992, 30, 473-483. [CrossRef]

36. Jawinski, P.; Kittel, J.; Sander, C.; Huang, J.; Spada, J.; Ulke, C.; Wirkner, K.; Hensch, T.; Hegerl, U. Recorded and reported sleepiness: The association between brain arousal in resting state and subjective daytime sleepiness. Sleep 2017, 40, zsx099. [CrossRef]

37. Hegerl, U.; Hensch, T. The vigilance regulation model of affective disorders and ADHD. Neurosci. Biobehav. Rev. 2014, 44, 45-57. [CrossRef] [PubMed]

38. Ulke, C.; Sander, C.; Jawinski, P.; Mauche, N.; Huang, J.; Spada, J.; Wittekind, D.; Mergl, R.; Luck, T.; Riedel-Heller, S.; et al. Sleep disturbances and upregulation of brain arousal during daytime in depressed versus non-depressed elderly subjects. World J. Biol. Psychiatry 2017, 18, 633-640. [CrossRef]

39. Ulke, C.; Wittekind, D.A.; Spada, J.; Franik, K.; Jawinski, P.; Hensch, T.; Hegerl, U. Brain arousal regulation in SSRI-medicated patients with major depression. J. Psychiatr. Res. 2019, 108, 34-39. [CrossRef]

(C) 2020 by the authors. Licensee MDPI, Basel, Switzerland. This article is an open access article distributed under the terms and conditions of the Creative Commons Attribution (CC BY) license (http://creativecommons.org/licenses/by/4.0/). 